\title{
The Effects of On-Demand Behavior in Routing Protocols for Multihop Wireless Ad Hoc Networks
}

\author{
David A. Maltz, Josh Broch, Jorjeta Jetcheva, and David B. Johnson, Member, IEEE
}

\begin{abstract}
A number of different routing protocols proposed for use in multi-hop wireless ad hoc networks are based in whole or in part on what can be described as on-demand behavior. By ondemand behavior, we mean approaches based only on reaction to the offered traffic being handled by the routing protocol. In this paper, we analyze the use of on-demand behavior in such protocols, focusing on its effect on the routing protocol's forwarding latency, overhead cost, and route caching correctness, drawing examples from detailed simulation of the dynamic source routing (DSR) protocol. We study the protocol's behavior and the changes introduced by variations on some of the mechanisms that make up the protocol, examining which mechanisms have the greatest impact and exploring the tradeoffs that exist between them.
\end{abstract}

Index Terms-Communication system routing, computer network performance, dynamic source routing (DSR) protocol, wireless ad hoc networks.

\section{INTRODUCTION}

$\mathbf{I}^{2}$ $\mathrm{N}$ a wireless ad hoc network, individual mobile nodes forward packets for other communicating mobile nodes that are out of the wireless transmission range of each other. The network is dynamically self-organizing and self-configuring, with nodes establishing the necessary routing between each other without requirement for any existing infrastructure or administration. Since communicating mobile nodes may be some distance apart, multiple network "hops" through intermediate mobile nodes may be required to extend the communication range between them. As communicating nodes or those between them that are forwarding packets move about, the routing protocol in use in the network must adapt its routing decisions to enable continued communication between the nodes. The rate of topology change, and thus the rate of routing protocol reaction, may be quite dramatic in some ad hoc networks.

Many different routing protocols for use in such networks have been proposed, utilizing a wide variety of different routing algorithms and approaches. A number of these proposed protocols are based in whole or in part on what can be described as on-demand behavior [1], [3], [5]-[7], [9], [10], [12]-[15], allowing them to dynamically adapt the level of routing protocol activity required to correctly handle the offered traffic. By on-demand behavior, we mean approaches

Manuscript received June 10, 1998; revised April 22, 1999. This work was supported in part by the NSF under CAREER Award NCR-9502725, in part by the Air Force Material Command under DARPA contract F19628-96-0061, and in part by the AT\&T Foundation under a Special Purpose Grant in Science and Engineering.

The authors are with the Computer Science Department, Carnegie Mellon University, Pittsburgh, PA 15213 USA (e-mail: dmaltz@cs.cmu.edu; broch@andrew.cmu.edu; jorjeta@cs.cmu.edu; dbj@cs.cmu.edu).

Publisher Item Identifier S 0733-8716(99)04805-2. based only on reaction to the presence of data packets. The use of strictly periodic or timer-based activities, such as typical router advertisements, link or neighbor status sensing messages, or the deletion of routing table or cache entries after some expiration time, are not considered here. Although these other approaches may be quite useful as part of protocols for routing in wireless ad hoc networks, our aim here is to analyze the use of on-demand behavior as found in a number of proposed protocols.

In this paper, we focus on three questions that apply in general to any wireless ad hoc network routing protocol using on-demand behavior.

- What effect does on-demand routing have on packet latency? An on-demand routing protocol attempts to discover a route to a destination only when it is presented with a packet for forwarding to that destination. This discovery must be completed before the packet can be sent, which adds to the latency of delivering the packet. Indeed, some mechanisms to reduce the overhead cost of discovering a new route may result in an increase in latency for some route discovery attempts.

- What is the overhead cost of on-demand routing behavior? Without additional information, a protocol using ondemand routing must search the entire network for a node to which it must send packets, but does not know how to reach. Optimizations to the protocol may reduce the cost of initiating communication, but discovering a new route is likely to remain a costly operation.

- When caching the results of on-demand routing decisions, what is the level and effect of caching and cache correctness on the routing protocol? Any on-demand routing protocol must utilize some type of routing cache in order to avoid the need to rediscover each routing decision for each individual packet. However, the cache itself may contain out-of-date information indicating that links exist between nodes that are no longer within wireless transmission range of each other. This stale data represents a liability that may degrade performance rather than improve it.

To address these questions, we utilize examples drawn from detailed simulation of the dynamic source routing (DSR) protocol [1], [9], [10], studying its behavior and the changes introduced by variations on some of the mechanisms that comprise it. The DSR protocol provides a good source of examples for this study, since it is based entirely on on-demand behavior, although we believe that our results here generalize to other protocols using related approaches. 
Previous simulation efforts have evaluated the performance of ad hoc routing protocols with respect to varying environmental conditions, such as the number of nodes, the nodes' movement pattern, or the traffic load. Evaluations of this form tend to examine only a few summary metrics of interest to network users (e.g., packet delivery ratio or routing overhead). In contrast, our goal is to dissect the protocol into its component mechanisms. We seek to determine both the effectiveness of the individual mechanisms and the manner in which they interact to contribute to the overall performance measured by summary metrics.

In Section II of this paper, we give an overview of the operation of the DSR protocol, and in Section III, we discuss our simulation framework and methodology for this study. In Section IV, we give a summary of the baseline performance of DSR, including all optimizations to the protocol. The next three sections then present our analysis of each of the general questions posed earlier: Section V covers latency issues, Section VI covers overhead cost, and Section VII covers cache consistency. Finally, in Section VIII, we present conclusions and discuss future work.

\section{OVERVIEW OF THE DSR PROTOCOL}

The DSR protocol [1], [9], [10] is composed of two mechanisms: Route Discovery and Route Maintenance, each of which operates using entirely on-demand behavior. When a node in the ad hoc network attempts to send a packet to some destination, if it does not already know a route to that destination, it uses Route Discovery to dynamically discover one. The route is cached and used as needed for sending subsequent packets, each of which utilizes the Route Maintenance mechanism to detect if the route has broken, for example, due to two nodes along the route moving out of wireless transmission range of each other. Route Discovery is only invoked when needed, and Route Maintenance operates only when actively using the route to send individual packets.

The routes that DSR discovers and uses are source routes. That is, the sender learns the complete ordered sequence of network hops necessary to reach the destination, and each packet to be routed carries this list of hops in its header. The key advantage of a source routing design is that intermediate nodes do not need to maintain up-to-date routing information in order to route the packets that they forward, since the packets themselves already contain all the routing decisions. This fact, coupled with the on-demand nature of the protocol, eliminates the need for the periodic route advertisement and neighbor detection packets present in other protocols.

Route Discovery works by flooding a request through the network in a controlled manner, seeking a route to some target destination. In its simplest form, a source node $\mathbf{S}$ attempting to discover a route to a destination node $\mathbf{D}$ broadcasts a ROUTE REQUEST packet that is rebroadcast by intermediate nodes until it reaches $\mathbf{D}$, which then answers by returning a ROUTE REPLY packet to $\mathbf{S}$. Many optimizations to this basic mechanism are used to attempt to limit the frequency and spread of Route Discovery attempts.

When sending or forwarding a packet to some destination $\mathbf{D}$, Route Maintenance is used to detect if the network topology has changed such that the route used by this packet has broken. Each node along the route, when transmitting the packet to the next hop, is responsible for detecting if its link to the next hop has broken. In many wireless medium access control (MAC) protocols, such as IEEE 802.11 [8], the MAC protocol retransmits each packet until a link-layer acknowledgment is received or until a maximum number of transmission attempts have been made. Alternatively, DSR may make use of a passive acknowledgment [11] or may request an explicit network-layer acknowledgment. When the retransmission and acknowledgment mechanism detects that the link is broken, the detecting node returns a ROUTE ERROR packet to the original sender $\mathbf{S}$ of the packet. For subsequent data packets, the sender $\mathbf{S}$ can attempt to use any other route to $\mathbf{D}$ that is already in its route cache, or can invoke Route Discovery again to find a new route.

\section{A. Optimizations to Route Discovery}

Nonpropagating ROUTE REQUESTS: When performing Route Discovery, nodes first send a ROUTE REQUEST with the maximum propagation limit (hop limit) set to zero, prohibiting their neighbors from rebroadcasting it. At the cost of a single broadcast packet, this mechanism allows a node to query the route caches of all its neighbors for a route and optimizes the case in which the destination node is adjacent to the source. If the nonpropagating ROUTE REQUEST fails to elicit a reply within a $30 \mathrm{~ms}$ time limit, a propagating ROUTE REQUEST with a hop limit set to the maximum value is sent. The 30 ms time out was chosen based on the distribution of REPLY latencies shown in Fig. 4.

Replying from Cache: If a node receives a ROUTE REQUEST for a destination $\mathbf{D}$ to which it has a route, the node may generate a ROUTE REPLY based on its cached information instead of rebroadcasting the ROUTE REQUEST. This optimization is intended to both reduce the latency of ROUTE REPLIES and prevent ROUTE REQUESTS from flooding through the entire network.

Gratuitous ROUTE REPLIES: When a node overhears a packet not addressed to itself, the node checks if the packet's header contains its address in the unprocessed portion of the source route. If so, the node knows that packet could bypass the unprocessed hops preceding it in the source route. The node then sends a gratuitous ROUTE REPLY message to the packet's source, giving it the shorter route without these hops. Upon receiving the ROUTE REPLY, the originator will insert the shorter route into its route cache. The route cache can store more than one route to a destination, so the shorter route will not necessarily overwrite the longer route already in the cache. If the shorter route is found not to work, the originator can immediately revert to the longer route.

\section{B. Optimizations to Route Maintenance}

Salvaging: When an intermediate node forwarding a packet discovers that the next hop in the source route for the packet is unreachable, it examines its route cache for another route to the same destination. If a route exists, the node replaces the broken source route in the packet's header with the route 
from its cache and retransmits the packet. If a route does not exist in its cache, the node drops the packet-it does not send a Route REQuEST. In either case, the node attempting to perform salvaging returns a ROUTE ERROR to the source of the data packet.

Gratuitous ROUTE ERRORS: When a source $\mathbf{S}$ receives a ROUTE ERROR for a packet that it originated, $\mathbf{S}$ propagates this ROUTE ERROR to its neighbors by piggybacking it on its next RoUTE REQUEST. In this way, stale information in the caches of nodes around $\mathbf{S}$ will not generate ROUTE REPLIES that contain the same invalid link for which $\mathbf{S}$ received a RoUTE ERROR.

\section{Optimizations to Caching Strategies}

Snooping: When a node forwards a data packet, it "snoops" on the unprocessed portion of the source route and adds to its cache the route from itself to the final destination listed in the source route.

Tapping: Nodes operate their network interfaces in promiscuous mode, disabling the interface's address filtering and causing the network protocol to receive all packets that the interface overhears. These packets are scanned for useful source routes or ROUTE ERROR messages and then discarded. This optimization allows a node to prime its route cache with potentially useful information, while causing no additional use of the limited network bandwidth.

\section{METHODOLOGY}

We analyzed the effect of the mechanisms that comprise DSR by simulating different variations of the DSR protocol on an identical network with an identical workload. Simulation enabled us to study a large number of points in the DSR design space and to directly compare the results of the simulations, since we were able to hold constant factors such as the communication and movement pattern between runs of the simulator. We conducted the experiments using the $n s-2$ network simulator [4] extended with our support for realistic modeling of mobility and wireless communication [2]. The simulator allows the specification of arbitrary movement patterns for the nodes and correctly models the effects of contention for the media and the distance between nodes in determining whether a transmitted packet will be successfully received. Each simulation used 50 nodes and simulated 900 seconds of real time.

Each node in the simulation communicated via a radio with the characteristics of the Lucent Technologies WaveLAN product [16]. WaveLAN is a shared-media radio with a raw capacity of $2 \mathrm{Mbit} / \mathrm{s}$ and a $250 \mathrm{~m}$ nominal range. The exact range of a transmission varies with the number of simultaneous transmissions since our simulator models the attenuation of transmitted signals with distance and capture effects. At the link layer, we simulate the complete distributed coordination function (DCF) MAC protocol of the IEEE 802.11 Wireless LAN standard [8].

\section{A. Node Movement and Communication Pattern}

In preparing this paper, we analyzed the effect of two different movement models: constant node motion and no node motion. All tables and graphs in this paper are from the constant motion case, since without motion, DSR does a single Route Discovery for each destination, and then aside from congestion-caused packet drops leading to ROUTE ERRORS, there is no more protocol activity for the duration of the run. For both constant motion and no motion models, the nodes start at a uniformly distributed location. In the constant motion case, all nodes then move according to the Random Waypoint algorithm [10], [2] wherein each node picks a new random location in the simulated area and proceeds there at a speed chosen uniformly from zero to $20 \mathrm{~m} / \mathrm{s}$. When the node reaches this waypoint, it picks another location to move to and repeats the cycle.

We chose to model node communication using a uniform node-to-node communication pattern with constant bit rate (CBR) traffic sources sending data in 512-byte packets at a rate of four packets/s. A total of $20 \mathrm{CBR}$ connections were modeled in each simulation run, with each node being the source of zero, one, or two connections. In all runs, the 20 connections were spread in this way over a total of 14 different originating nodes (which we label nodes 1-14). All CBR connections were started at times uniformly distributed during the first $180 \mathrm{~s}$ of simulated time and then remained active through the entire simulation. We chose the parameters of the communication pattern to stress the ability of the routing protocol to discover and maintain routes during the experiments, but to avoid causing extreme congestion.

In order to average out the effects of particular motion or communication patterns, we generated ten different scenarios-five with constant node motion and five with no node motion. We ran all experiments over the same set of scenarios.

\section{B. Simulated Sites}

In an attempt to generate results that would be representative of some potential real-world scenarios that DSR might encounter, we ran our simulations over two different types of simulated sites:

- the rectangular site with length much greater than its width and dimensions of $1500 \times 300 \mathrm{~m}$;

- the square site with equal length and width and dimensions of $670 \times 670 \mathrm{~m}$ so the area is approximately equal to that of the rectangular site.

Altering the shape of the simulated site in this way creates networks with qualitatively different topologies and throughput bottlenecks, thereby exercising DSR in different ways.

The rectangular site causes a roughly linear arrangement of the nodes. The results seen here could be applied to situations where the physical paths between nodes are very constrained, such as when the mobile nodes are vehicles driving along a road. The lengths of the routes taken by packets in this site are typically longer than the corresponding route lengths in the square site. Since the site is also narrow, a packet being transmitted down the site is typically overheard by all the nodes spatially located between the sender and destination. This makes networks in the rectangular site more prone to congestion bottlenecks since there is little spatial diversity in the narrow dimension. 


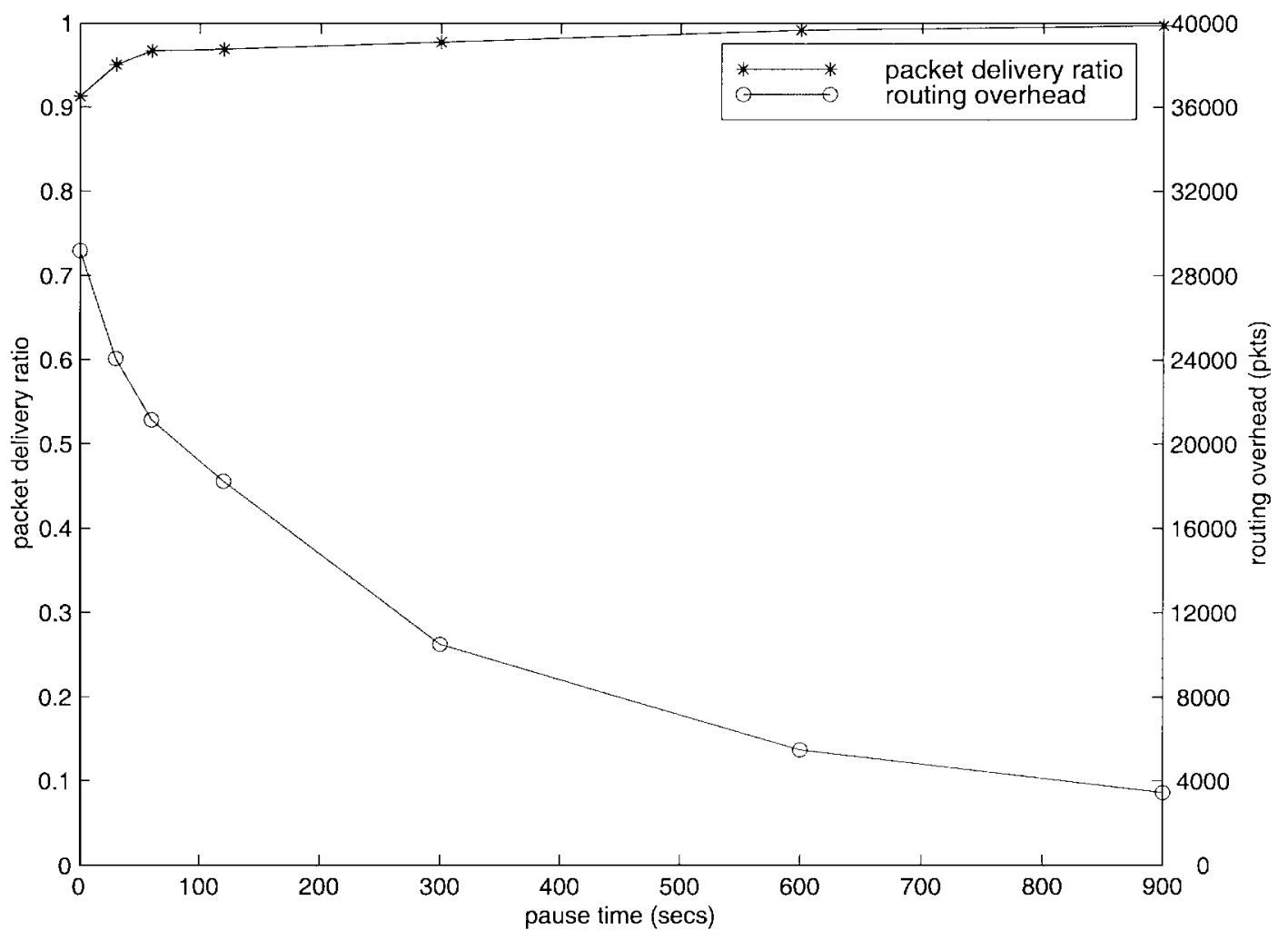

Fig. 1. Baseline DSR packet delivery ratio and routing packet overhead (all-opt DSR, rectangular site).

The square site models situations in which nodes can move freely around each other and where there is a reasonable amount of path and spatial diversity available for the routing protocol to discover and use. Since its total area is approximately the same as that of the rectangular site, the average node density between the two sites is constant, but the average route length used in the square site is less than in the rectangular site.

\section{BASELINE EVALUATION OF DSR}

In order to characterize the performance of DSR in the scenarios used for this paper, we evaluated the performance of DSR based on the following two metrics:

- packet delivery ratio: the ratio between the number of packets originated by the "application layer" CBR sources and the number of packets received by the CBR sinks at the final destinations;

- routing overhead: the total number of routing packets transmitted during the simulation; for packets sent over multiple hops, each transmission of the packet (each hop) counts as one transmission.

Packet delivery ratio is important as it describes the loss rate that will be seen by the transport protocols, which in turn determines the maximum throughput that the network can support. This metric characterizes both the completeness and correctness of the routing protocol.

The routing overhead metric is important because it measures the scalability of a protocol, the degree to which it will function in congested or low-bandwidth environments, and its efficiency in terms of consuming node battery power. A protocol that sends large numbers of routing packets can also increase the probability of packet collisions and may delay data packets in network interface transmission queues.

Fig. 1 shows the packet delivery ratio (the top curve on the graph) and routing packet overhead (the bottom curve) for the all-opt DSR version of the protocol, which utilizes all of the optimizations described in Section II. Both metrics are plotted as a function of the node mobility rate, measured in seconds of pause time [2], with $0 \mathrm{~s}$ meaning constant node motion and $900 \mathrm{~s}$ meaning no node motion. All-opt DSR is able to deliver $90 \%$ or more of the data packets originated by the CBR traffic sources, and the routing overhead scales well with pause time, remaining reasonable even at constant node mobility. The offered traffic load and movement pattern in these scenarios was the same as that described in Section III.

The figure confirms our prior experience comparing four ad hoc network routing protocols [2], which showed that DSR has great potential as an efficient protocol in a multihop ad hoc network environment. However, DSR is only one example of the larger class of on-demand routing protocols. The remainder of this paper is a detailed analysis of several of DSR's mechanisms, aimed at identifying their strengths and weaknesses, in order to guide the design of future on-demand protocols.

\section{EFFECTS ON LATENCY}

The use of on-demand behavior in routing protocols for multihop wireless ad hoc networks can result in increased packet latency due to the need to delay sending a packet if the packet requires a route to a previously unknown destination. When some node $\mathbf{A}$ wants to send a packet to a node $\mathbf{B}$, and it does 


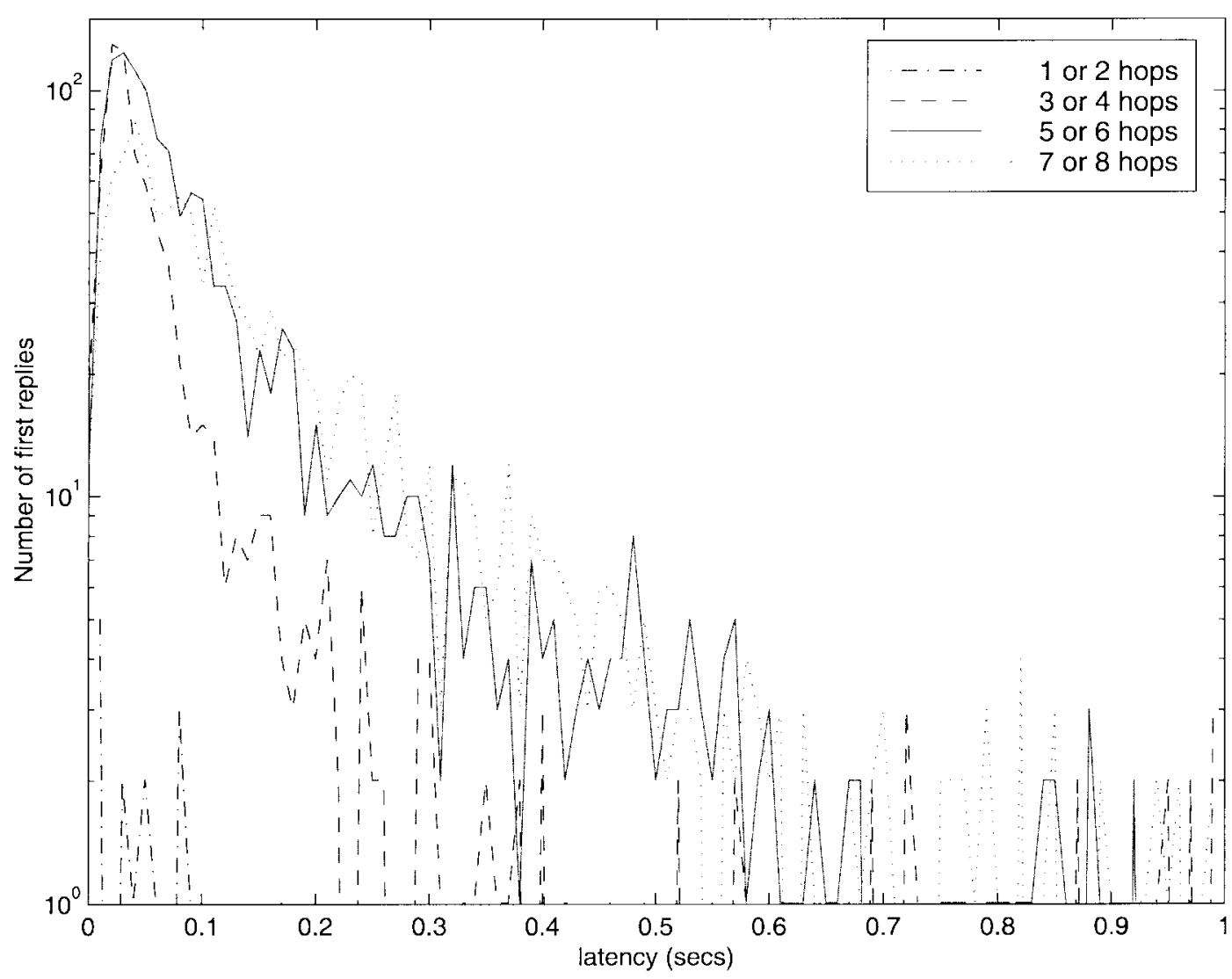

Fig. 2. Distribution of latencies of RouTE REPLY packets by number of hops (no cache replies, rectangular site, constant motion).

not currently have a route to $\mathbf{B}$, it must first perform a Route Discovery for node $\mathbf{B}$ before sending the packet. Because A must buffer its packet until it has a route to $\mathbf{B}$, the entire time that Route Discovery takes to obtain a route to $\mathbf{B}$ adds directly to the time it will take for the packet to be delivered. This makes the latency of Route Discovery critical in any environment in which packets must be delivered in a timely fashion.

In the DSR protocol, each Route Discovery consists of two phases.

- Nonpropagating Route ReQuest: node A transmits a ROUTE REQUEST with a maximum propagation limit of zero. Any node receiving the RouTE REQUEST that does not have a route to $\mathbf{B}$ simply discards the request.

- Propagating Route ReQuest: if after $30 \mathrm{~ms}$ the nonpropagating ROUTE REQUEST has failed to return a route to $\mathbf{B}$, node $\mathbf{A}$ will then transmit a propagating ROUTE REQUEST. Each node other than $\mathbf{B}$ that receives this request will either return a ROUTE REPLY based on information in its route cache or will rebroadcast the ROUTE REQUEST, propagating it further through the network. Should B itself receive the request, it will return a ROUTE REPLY consisting of the source route collected in the ROUTE REQUEST.

We consider two aspects of latency: the amount of time it takes a node to acquire a route to a destination and the amount of time it takes a sender to "recover" (find a new source route) when a route that it is using breaks. In order to calculate the latency of a Route Discovery, we measure the time from when a node sends a ROUTE REQUEST until it receives the first RoUTE REPLY that answers the request. We use the first reply to calculate latency since as soon as it arrives, the node can begin transmitting data packets-it need not wait for multiple replies to be returned before sending.

The individual per-hop forwarding latency of all packets is dictated by the length of the interface queue and by the media access time, since the packets at each node must wait their turn for transmission. The latency of a single ROUTE REQUEST propagating outwards or a ROUTE REPLY propagating inwards is dominated by effects due to path length: the greater the number of hops over which the ROUTE REQUEST or ROUTE REPLY packet must travel, the longer the time the packet takes to reach its destination. However, the greatest influence on the latency of a Route Discovery is the distribution of information in nodes' caches, since it is the path length to the closest node that can generate a RouTE REPLY that determines the latency of Route Discovery.

The latency of the slowest Route Discoveries seen during an experiment are dominated by the effects of congestion. The maximum latencies reported in this section are frequently in the tens of seconds-we have verified in each case that the REQUEST or REPLY was held up in a series of long interface queues as the packet propagated, each queue draining very slowly due to media contention around the node.

\section{A. Latency Related to Path Length}

In Fig. 2, we show the distribution of times required for Route Discovery, broken out by the number of hops in the discovered route. Fig. 3 is based on the same data, showing 


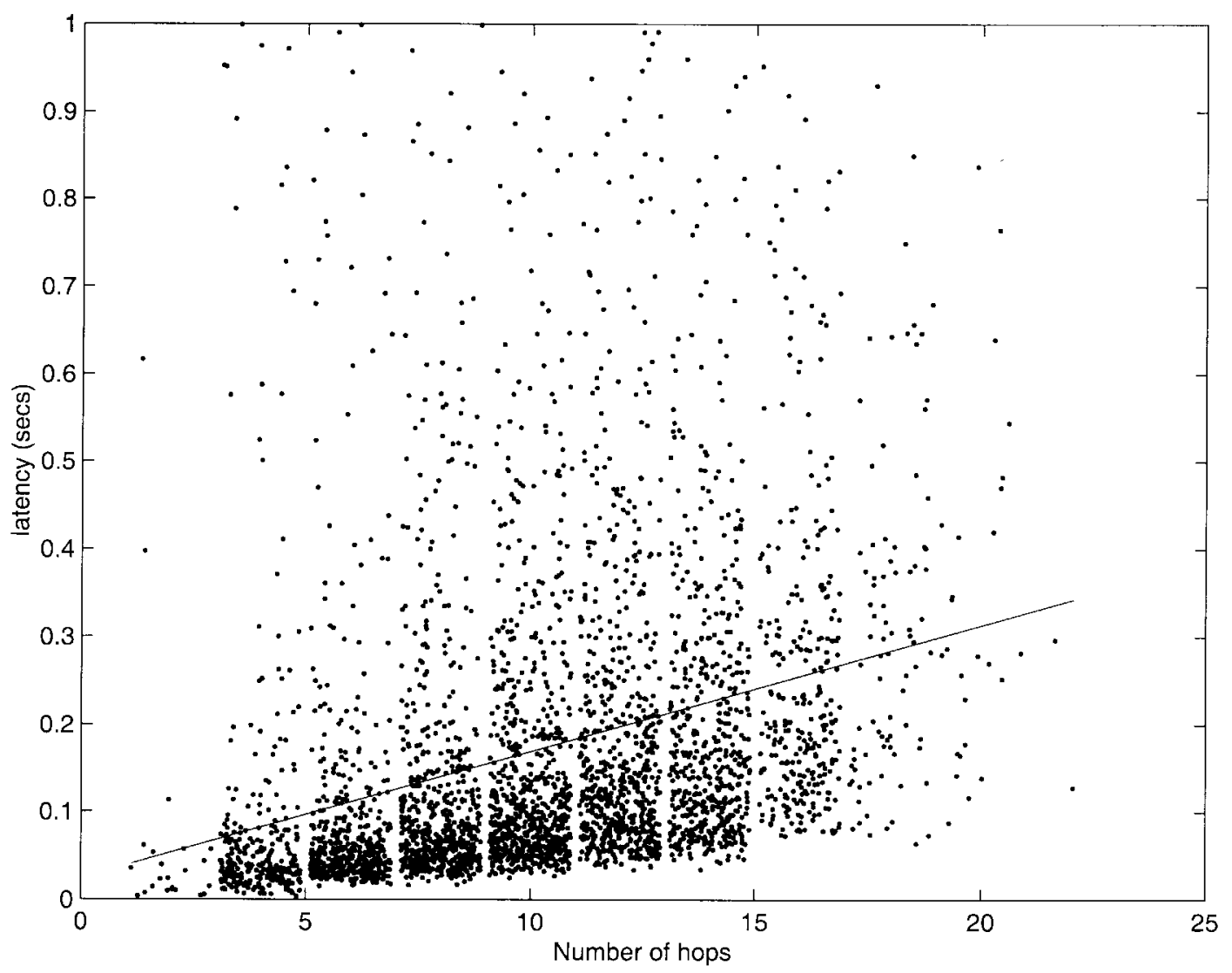

Fig. 3. Scatter plot of forwards versus latency. Forwards includes the number of hops from the originator of the Route Discovery to the target and from the target back to the originator (no cache replies, rectangular site, constant motion).

the latency of Route Discovery as a scatter plot versus the number of hops over which the ROUTE REQUEST and matching ROUTE REPLY had to travel. The scatter plot makes visible the distribution of individual samples, and the abscissas have been uniformly jittered to make the density of the points visible. The least squares fit the line shown on the graph has a slope of $14.5 \mathrm{~ms} / \mathrm{hop}$.

The spread of latencies recorded reflects the realistic nature of the simulator used. The minimum time required to forward a small packet, such as a ROUTE REQUEST or ROUTE REPLY, over a single hop is approximately $600 \mu$ s. This assumes the packet immediately acquires the media when offered to the network. The minimum per-hop forwarding time for a 512byte data packet is approximately $3 \mathrm{~ms}$. However, since the effective carrier-sense range on the simulated radios is $550 \mathrm{~m}$, on average, each packet sent must defer to other packets being transmitted elsewhere in the network.

\section{B. Latency of Route Discovery}

The ROUTE REPLY packets received by a node in response to sending a ROUTE REQUEST may be categorized as follows.

- Cache replies: replies constructed from cached routing information by a node other than the target of a Route Discovery.

- Target replies: replies originated by a node in response to receipt of a ROUTE REQUEST targeting it-we say
TABLE I

Latency of First Route RePly by Type (All-Opt DSR, Rectangular Site, Constant Motion)

\begin{tabular}{l|c|c|c|c|c}
\cline { 3 - 6 } & \multicolumn{4}{c}{} & \multicolumn{4}{c}{ Latency } \\
\cline { 2 - 6 } & \# Replies & Mean & Min & 99 percentile & Max \\
\hline Neighbor Replies & 3136 & $7.1 \mathrm{~ms}$ & $1.3 \mathrm{~ms}$ & $457 \mathrm{~ms}$ & $17.8 \mathrm{~s}$ \\
\hline Cache Replies & 1524 & $45.9 \mathrm{~ms}$ & $1.3 \mathrm{~ms}$ & $752 \mathrm{~ms}$ & $2.4 \mathrm{~s}$ \\
\hline Target Replies & 12 & $87.6 \mathrm{~ms}$ & $23.6 \mathrm{~ms}$ & $458 \mathrm{~ms}$ & $458.3 \mathrm{~ms}$ \\
\hline
\end{tabular}

that target replies are based on fresh routing information because the route from requester to target has just been traversed by the corresponding ROUTE REQUEST.

- Neighbor replies: a RoUTE REPLY returned in response to a ROUTE REQUEST with a maximum propagation limit (TTL) of zero-neighbor replies must originate from a direct neighbor of the requester (since the request cannot propagate), but may be either cache replies or target replies depending on whether or not the respondent is the target of the Route Discovery.

Table I summarizes the latency required for the initiator of a Route Discovery to receive the first ROUTE REPLY in response to its Route ReQuest. This data is broken down into the mean, minimum, and maximum latencies for each of the three different types of ROUTE REPLIES listed earlier. In this section, we analyze the latency for each type of ROUTE REPLY and examine the effect of changing the shape of the site on the latency. 


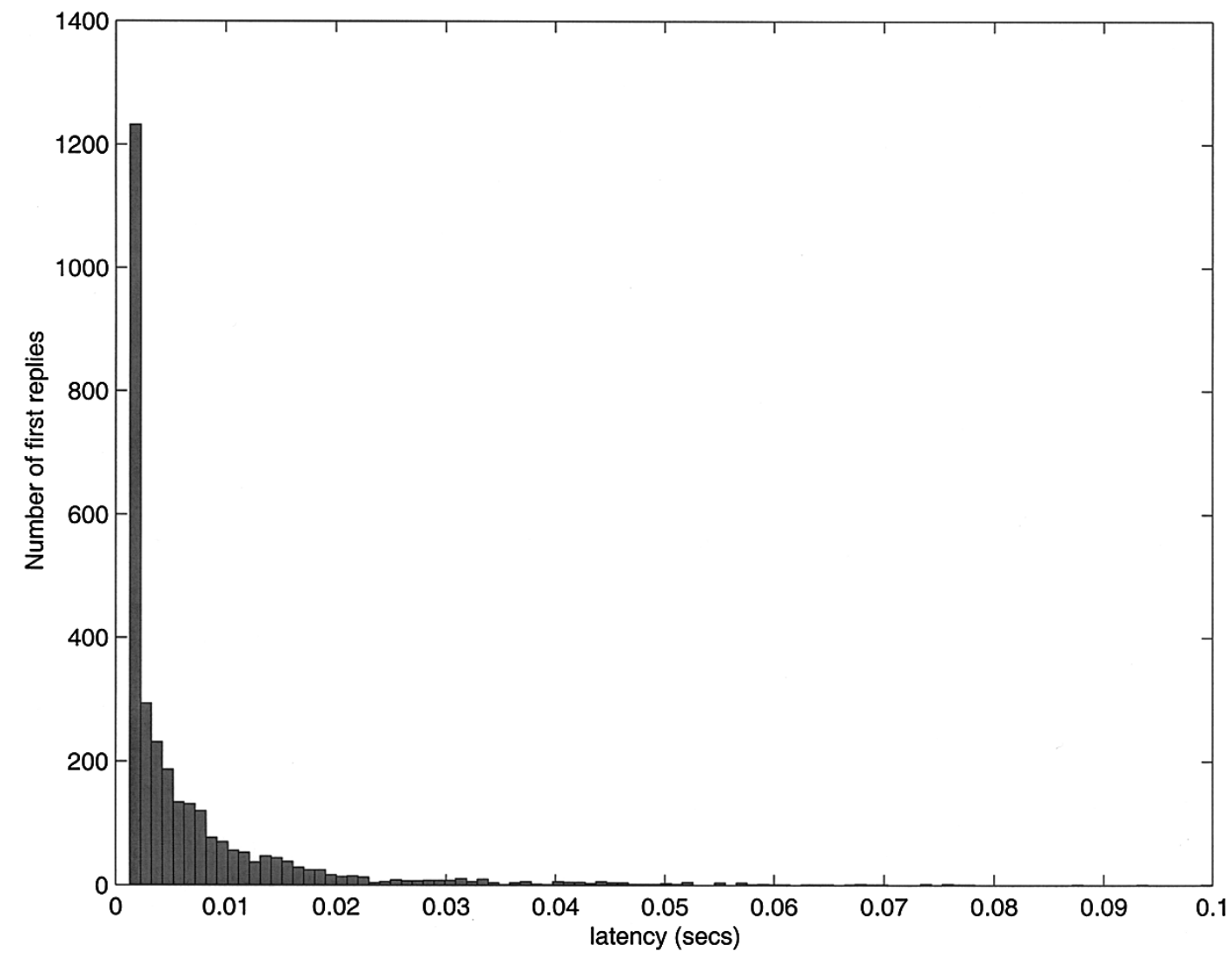

Fig. 4. Latency of neighbor replies (all-opt DSR, rectangular site, constant motion).

1) The Latency of Neighbor Replies: Fig. 4 shows a detailed view of the latency of the first ROUTE REPLY packet received for nonpropagating ROUTE REQUESTS. The graph shows only those replies that were returned within $100 \mathrm{~ms}$. After $30 \mathrm{~ms}$, a nonpropagating request is considered unsuccessful, and a propagating ROUTE REQUEST is transmitted, and so only replies returned within the $30 \mathrm{~ms}$ time out are useful in terms of preventing a propagating Route Discovery. Since neighbor replies with latency greater than $30 \mathrm{~ms}$ may be interpreted as the first reply to the propagating ROUTE REQUEST, we have included replies with a latency of up to $100 \mathrm{~ms}$ in our analysis. The mean of this distribution (Table I) is $7.1 \mathrm{~ms}$ and was computed by discarding as outliers all replies that arrived after $100 \mathrm{~ms}$.

2) The Latency of Cache Replies: When a nonpropagating request fails to obtain a route, the node performing Route Discovery sends a propagating RouTE REQUEST. Each node that hears this request will either transmit a ROUTE REPLY from its cache or forward the request as described earlier. Fig. 5 shows a detailed view of the latency of ROUTE REPLY packets sent from cached route information received in response to a propagating RouTE REQUEST. Again discarding outliers, the mean of this distribution is $45.9 \mathrm{~ms}$ (Table I), nearly seven times larger than the mean latency for neighbor replies (7.1 $\mathrm{ms})$. This difference between mean neighbor reply latency and the latencies of cache replies indicates that successful nonpropagating requests are very effective in reducing latency. However, the difference does not show exactly how effective they are since the cache replies in Table I are elicited by propagating requests that are only sent after a nonpropagating request has already failed.

In order to factor out the effect of nonpropagating ROUTE REQUESTS on the latency of cache replies, we removed the mechanism that sends nonpropagating requests and ran this modified version of DSR on the same set of scenarios. The results of this experiment are reported in Table II and Fig. 6. When only propagating ROUTE REQUESTS are sent, the mean latency for cache replies dropped to $21 \mathrm{~ms}$, less than half of the latency measured when nonpropagating requests were enabled.

3) The Latency of Target Replies: The third row of Table I in Section V-B summarizes the latency of replies from the target of a Route Discovery. The mean of $87.6 \mathrm{~ms}$ is an increase of approximately $40 \mathrm{~ms}$ over the time for cache replies. This increased latency is in general due to the increased number of hops over which the ROUTE REQUEST and matching ROUTE REPLY must propagate to reach the target, relative to what is needed to reach the first node with a route cache entry that can return a reply from its cache.

In almost all cases, the first ROUTE REPLY received in response to a ROUTE REQUEST is not a target reply, but rather is a cache reply. In our simulations, the first ROUTE REPLY came from the target node only a total of 12 times over all all-opt DSR scenarios studied. This small number of target replies indicates that DSR's caching mechanism and its ability to send replies from the cache are very effective in reducing the latency of Route Discovery, but this limits our ability to 


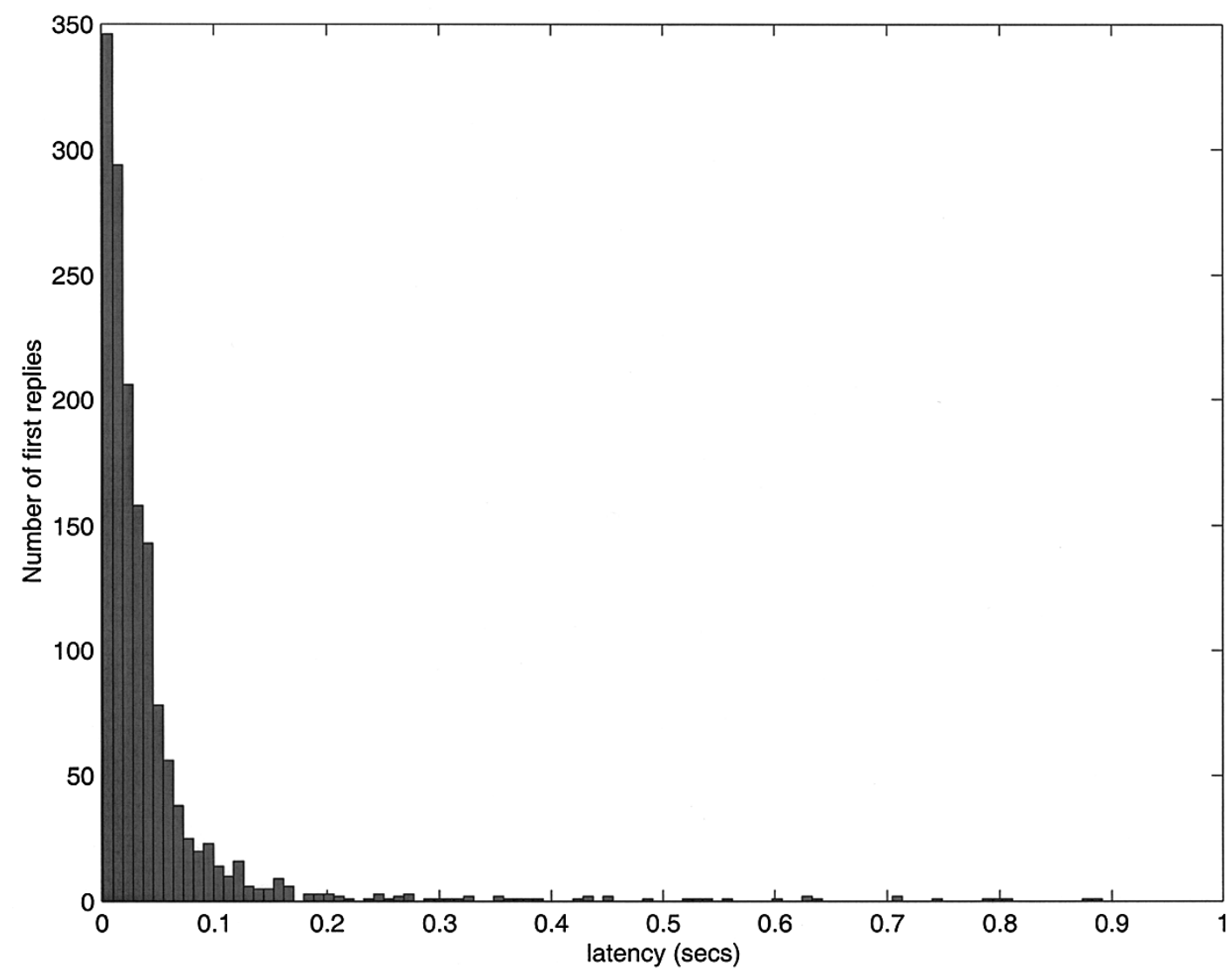

Fig. 5. Latency of Cached Route REPLY packets (all-opt DSR, rectangular site, constant motion).

TABLE II

Latency of First Route Reply (No NonPropagating Route Requests, Rectangular Site, Constant Motion)

\begin{tabular}{c|c|c|c}
\cline { 2 - 4 } & Mean Latency & Min Latency & Max Latency \\
\hline Cache Replies & $21.0 \mathrm{~ms}$ & $1.3 \mathrm{~ms}$ & $8.0 \mathrm{~s}$ \\
\hline Target Replies & $33.3 \mathrm{~ms}$ & $3.8 \mathrm{~ms}$ & $63.0 \mathrm{~ms}$ \\
\hline
\end{tabular}

directly evaluate the latency of target replies, and the latency for target replies shown in Table I represents only these 12 samples.

As another way of measuring the latency of target replies, we disabled both nonpropagating RoUTE REQUESTS and replying from cache in DSR and ran this modified version of the protocol over the same scenarios as above. Table III summarizes the target reply latencies from this experiment, and Fig. 7 shows a detailed view of this latency. The mean latency for target ROUTE REPLIES in this experiment (the only type enabled) was $403.1 \mathrm{~ms}$. This indicates that the optimizations added to DSR (replies from cache and nonpropagating route requests) significantly decrease the latency of Route Discovery. The reason latency is higher with the optimizations turned off is the decreased containment (Section VI) of ROUTE REQUESTS and the longer paths that ROUTE REPLIES and REQUESTS must take.

4) The Effect of a Different Topology: In the square site, we expect two factors to work together to decrease the latency of Route Discovery as compared to the rectangular site. The mean path length is less, so forwarding latency related to path length should be less, and the average degree of each node (the number of direct one-hop neighbors of each node) is higher, which should result in cache information being better distributed throughout the network, causing the first ROUTE REPLY received to come from nodes closer to the initiator of the Route Discovery.

The average degree of a node in the square site is 16.5 , as compared to 11.5 in the rectangular site. As shown in Table IV, the mean first ROUTE REPLY latency for all three types of REPLIES decreases relative to the latencies in the rectangular site (Table I), supporting the hypothesis that there are more nodes "close" to the originator of a Route Discovery and that routing information is better spread out through the network.

\section{Latency Incurred by Broken Routes}

As described in Section II, a node originating packets will detect that a route it is using has broken only after it tries to send a packet along the broken route and receives a ROUTE ERROR in response. The ROUTE ERROR is sent by the node that, upon attempting to forward the packet over a link in the source route, decides that the link is broken. The forwarding node declares the link broken after making several attempts to transmit the packet to the next hop and failing to receive a passive or explicit acknowledgment of success.

An important aspect of latency in the protocol is the time that it takes a source node $\mathbf{S}$ to detect that such a link in its route to a destination $\mathbf{D}$ has broken. This latency is a function of the time required to send the original packet (the packet 


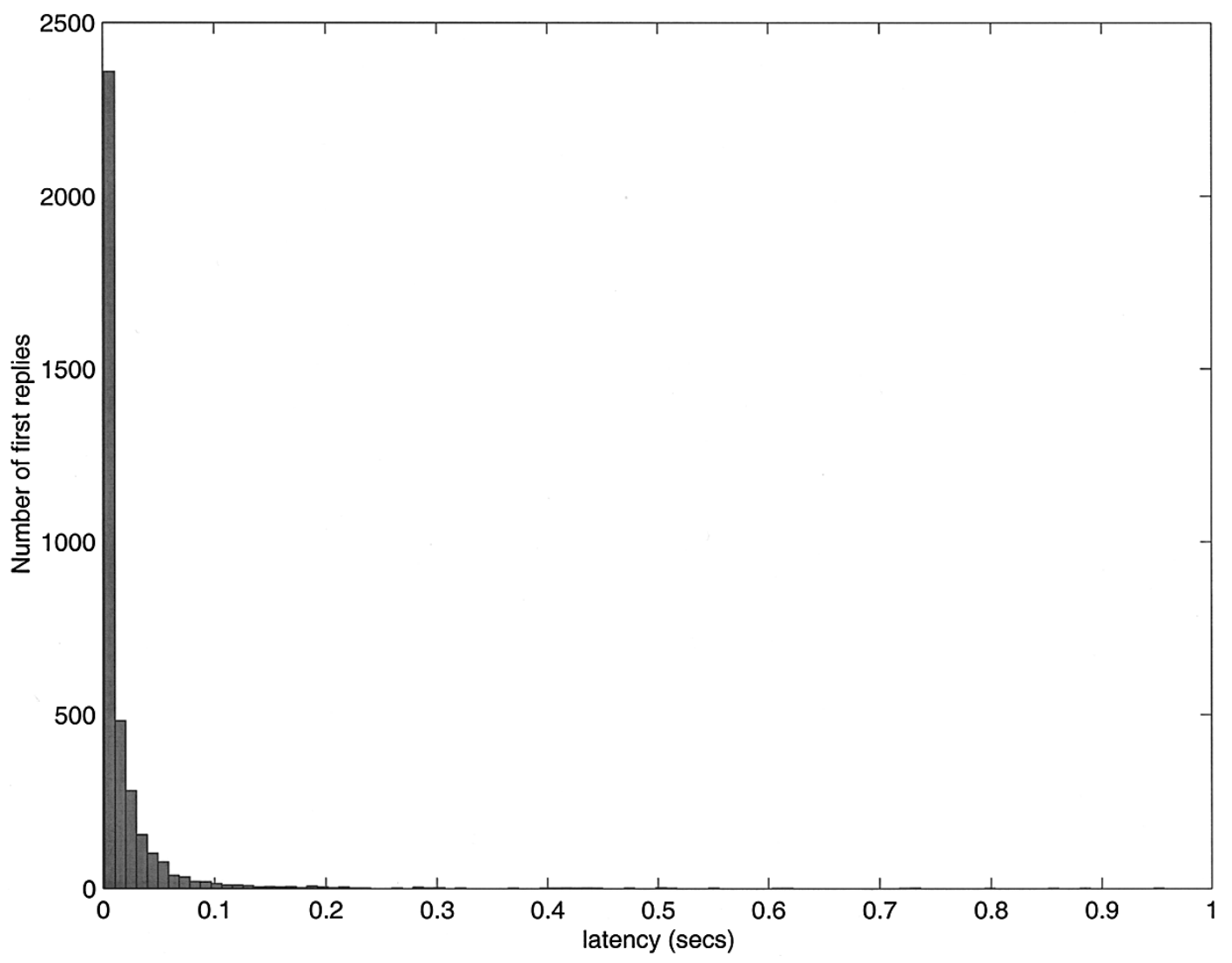

Fig. 6. Latency of cached RouTE REPLY packets (no nonpropagating RouTE REQUESTS, rectangular site, constant motion).

TABLE III

Latency of First Route Reply (No Nonpropagating Route ReQuests and No Cache Replies, Rectangular Site, Constant Motion)

\begin{tabular}{c|c|c|c}
\cline { 2 - 4 } & Mean Latency & Min Latency & Max Latency \\
\hline Target Replies & $403.1 \mathrm{~ms}$ & $3.5 \mathrm{~ms}$ & $42.1 \mathrm{~s}$ \\
\hline
\end{tabular}

triggering the ROUTE ERROR) along the route as far as the node that detects the broken link and the time required for that node to send the ROUTE ERROR back to the original sender.

For all-opt DSR in the rectangular site, Table V shows the time taken for a ROUTE ERROR to propagate from the node that detected a link breakage to the originator of the packet that contained the broken source route. The median latency per hop is $8.6 \mathrm{~ms}$, while the mean is $26.6 \mathrm{~ms}$. The difference between the two is due to the heavy tail caused by congestion. The average link breakage in this environment occurred 1.8 hops from the packet's originator.

\section{EFFECTS ON OVERHEAD COST}

Although on-demand routing protocols can reduce routing overhead by not disseminating routing information throughout the network on a periodic basis, the actual cost of performing on-demand Route Discovery can be significant. When a node A transmits a RouTE REQUEST, the request flood fills through the network, potentially disturbing each node in the network and consuming valuable bandwidth and battery power. Each node that receives the request must either transmit a ROUTE
REPLY based on information in its route cache or forward the request further.

We define two metrics to evaluate the cost of on-demand Route Discovery, which we refer to as containment and discovery cost.

- Containment is defined as the percentage of nodes that do not receive a particular RoUTE REQUEST. For a nonpropagating ROUTE REQUEST (Section II), containment is equivalent to measuring the percentage of nodes in the network that are not neighbors (within transmission range) of the node originating the request. For a propagating ROUTE REQUEST, containment measures how far out the request propagates before running into either the edge of the network or a band of nodes with cached information about the target that is wide enough to stop further propagation. Values of containment approaching one indicate that a ROUTE REQUEST was well contained and interrupted very few nodes, whereas containment values approaching zero indicate that most of the nodes in the network had to process the request.

- The cost of a single Route Discovery is defined as $1+$ FwReq + OgRep + FwRep, where 1 represents the transmission of the original request, FwReq is the number of Route REQuests forwarded, OgRep is the number of ROUTE REPLY originations, and FwRep is the number of ROUTE REPLIES forwarded. For each Route Discovery, this metric measures the number of routing packets (requests and replies) that were transmitted to complete 


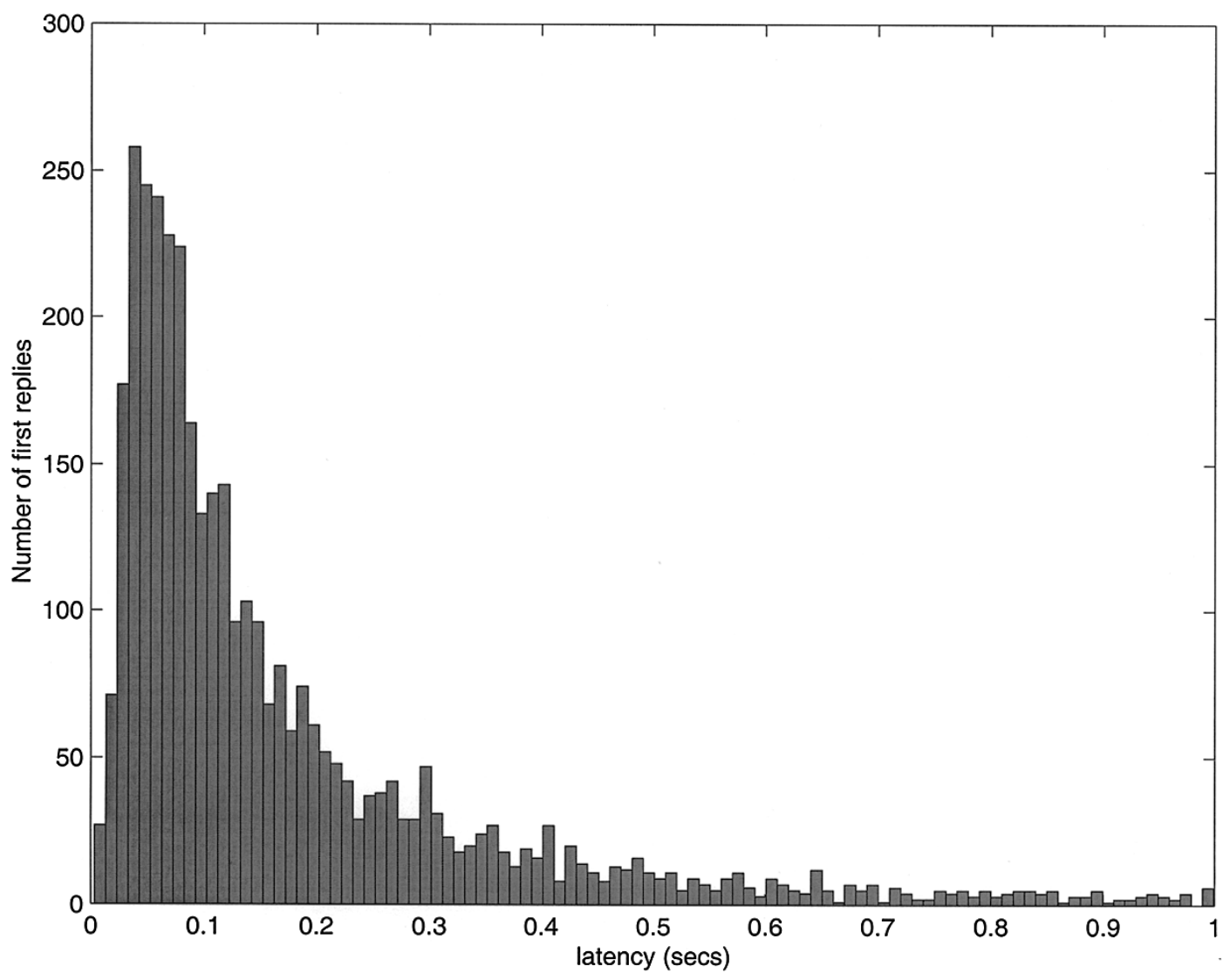

Fig. 7. Latency of Route REPLY packets (no nonpropagating RouTE REQUESTS and no cache replies, rectangular site, constant motion).

TABLE IV

Latency of First Route RePly by TyPe (All-Opt DSR, Square Site, Constant Motion)

\begin{tabular}{l|c|c|c}
\cline { 2 - 4 } & Mean Latency & Min Latency & Max Latency \\
\hline Neighbor Replies & $4.7 \mathrm{~ms}$ & $1.3 \mathrm{~ms}$ & $117.1 \mathrm{~ms}$ \\
\hline Cache Replies & $29.1 \mathrm{~ms}$ & $1.3 \mathrm{~ms}$ & $1.3 \mathrm{~s}$ \\
\hline Target Replies & $36.6 \mathrm{~ms}$ & $29.4 \mathrm{~ms}$ & $54.4 \mathrm{~ms}$ \\
\hline
\end{tabular}

TABLE V

LATENCY OF Route ERRORS (AlL-Opt DSR, Rectangular Site, Constant Motion)

\begin{tabular}{l|c|c|c|c|c|c|c|c}
\cline { 2 - 10 } & \multicolumn{8}{c}{ number of hops RoUTE ERROR travelcd } \\
\cline { 2 - 10 } & 1 & 2 & 3 & 4 & 5 & 6 & 7 & 8 \\
\hline median (ms) & 8.75 & 17.37 & 24.64 & 29.21 & 37.75 & 80.82 & 58.13 & 336.1 \\
\hline \# ERRORS & 8602 & 3985 & 1978 & 929 & 367 & 123 & 18 & 4 \\
\hline
\end{tabular}

the discovery. The average discovery cost is calculated as $\left(\mathrm{OgReq}+\sum \mathrm{FwReq}+\sum \mathrm{OgRep}+\sum \mathrm{FwRep}\right) / \mathrm{OgReq}$, where OgReq is the number of ROUTE REQUEST originations, and FwReq, and FwRep are summed over all Route Discoveries.

Our intuitive model of how Route Discovery works predicts that both the containment and discovery cost metrics of Route Discovery should be sensitive to the average degree of the nodes in the network. The degree of a node is the number of direct neighbors the node has and measures how tightly interconnected the network is. As the degree of interconnectivity goes up, it is harder to contain a ROUTE REQUEST to one part of the network. In addition, the "branching factor" of a propagating ROUTE REQUEST increases, which causes more nodes to receive and process it. Thus, we expect containment to decrease and discovery cost to increase in environments where the average node degree increases.

Using the containment and discovery cost metrics, we examine the overall cost of Route Discovery in DSR. We focus on how the use of route caches and nonpropagating requests effect Route Discovery among nodes in the rectangular site and then compare those results with data collected in the square site.

\section{A. Overall Route Discovery Cost in All-Opt DSR}

We began by studying the behavior of Route Discovery in scenarios using the rectangular site, where the average node degree was measured to be 11.5 neighbors. Route Discovery behaved as described in Sections II and V, with all-opt DSR sending a nonpropagating ROUTE REQUEST before transmitting a propagating ROUTE REQUEST if the nonpropagating request failed to provide a route within $30 \mathrm{~ms}$. Table VI summarizes the discovery costs of propagating and nonpropagating requests, broken out by the number of times a routing packet of the given type was originated $(\mathrm{Og})$ or forwarded $(\mathrm{Fw})$. When propagating and nonpropagating requests are used together in all-opt DSR, the total containment for all requests is $68 \%$, and the average discovery cost metric is nearly 17 . The two types 
TABLE VI

Summary of Route Discovery Costs (All-Opt DSR, Rectangular Site, Constant Motion)

\begin{tabular}{l|c|c|c}
\cline { 2 - 4 } & Nonpropagating & Propagating & Total \\
\hline Request Og & 957 & 316 & 1,273 \\
\hline Request Fw & 0 & 6,115 & 6,115 \\
\hline Reply Og & 3,912 & 3,215 & 7,127 \\
\hline Reply Fw & 0 & 7,002 & 7,002 \\
\hline Containment & $77.0 \%$ & $41.0 \%$ & $68.0 \%$ \\
\hline Cost & 5.09 & 52.69 & 16.90 \\
\hline
\end{tabular}

TABLE VII

Summary of Route Discovery Costs (No Reply From Cache AND No Nonpropagating Route Requests, Rectangular Site, Constant Motion)

\begin{tabular}{l|c}
\cline { 2 - 2 } & Propagating \\
\hline Request Og & 871 \\
\hline Request Fw & 39,471 \\
\hline Reply Og & 8,413 \\
\hline Reply Fw & 39,554 \\
\hline Containment & $10 \%$ \\
\hline Cost & 102.52 \\
\hline
\end{tabular}

of ROUTE REQUESTS have very different behavior and costs, however, and so it is informative to analyze them separately.

An average of 316 propagating requests were initiated during each of the simulations. Examining only these requests, on average each request was forwarded 20 times and caused ten ROUTE REPLY packets to be returned, yielding a containment metric of $41 \%$. This means that most propagating requests involve a little more than half the nodes in the network. The average discovery cost of a propagating request was nearly 53 transmissions. The fact that the origination of a single propagating ROUTE REQUEST results in the transmission of more than 50 DSR packets, even when the containment metric is $41 \%$, indicates that Route Discovery has the potential to be a very expensive operation when not well contained.

In comparison to the propagating ROUTE REQUESTS, the nonpropagating requests have a containment metric of $77 \%$ and the average discovery cost drops to only five transmissions. A propagating request costs nearly ten times more than a nonpropagating request because the total cost of a nonpropagating request is the one transmission of the ROUTE REQUEST plus one transmission for each of the neighbors that generates a ROUTE REPLY.

\section{B. Replying from Cache}

As described in Section II-A, DSR contains Route Discoveries by allowing a node to short circuit the outward propagation of a ROUTE REQUEST packet when it has a route to the request's target in its route cache. In order to discern the effect of replying from cache on the cost of Route Discovery, we reran all the scenarios with a modified version of DSR where replying from cache was disabled.

As shown in Table VII, the overall containment metric decreases to $10 \%$ when replying from cache is turned off. The cost of each discovery likewise increases by more than a factor of 5-102 packet transmissions per Route Discovery.
TABLE VIII

Summary of Route Discovery Costs (No Nonpropagating Route Requests, Rectangular Site, Constant Motion)

\begin{tabular}{l|c}
\cline { 2 - 2 } & Propagating \\
\hline Request Og & 776 \\
\hline Request Fw & 8,812 \\
\hline Reply Og & 8,077 \\
\hline Reply Fw & 8,894 \\
\hline Containment & $58 \%$ \\
\hline Cost & 34.19 \\
\hline
\end{tabular}

The dramatic decrease in containment and increase in overhead argue strongly that allowing nodes to reply from their route cache is vital to limiting the cost of discovery. Even though the discovery cost quintupled, the packet delivery ratio of DSR with cache replies disabled was the same as in all-opt DSR. While this is an encouraging result for on-demand Route Discovery, this may not be significant, as a different offered traffic load or communication pattern could suffer more from the dramatic increase in overhead.

\section{Nonpropagating Route Requests}

A second mechanism that may be used to reduce the cost of Route Discovery is an expanding ring search. Specifically, we consider the algorithm used by all-opt DSR where each propagating ROUTE REQUEST is preceded by a nonpropagating ROUTE REQUEST, i.e., with a maximum propagation limit (TTL) of zero. To evaluate whether the nonpropagating ROUTE REQUEST accomplishes its intended purpose of allowing the initiator of the Route Discovery to quickly and inexpensively query the route caches of each of its neighbors, we experimented with a version of DSR in which the sending of nonpropagating requests has been disabled.

When DSR is modified to send only propagating ROUTE REQUESTS (Table VIII), the overall containment metric decreases from $68 \%$ to $58 \%$, and the cost of a single Route Discovery doubles from 16 to 34 packets. These data argue strongly in favor of a two-phase Route Discovery that uses both nonpropagating and propagating requests, even though the tradeoff is an increase in latency when the nonpropagating request fails (Section V).

\section{Effects of Node Degree on Route Discovery}

As previously mentioned, nodes in the rectangular site have an average node degree of 11.5. To evaluate the effect of node degree on Route Discovery, we performed the same experiments using the square site where nodes have an average node degree of 16.5. This increase in the number of neighbors stems from the fact that the rectangular site is only $300 \mathrm{~m}$ wide, and hence, much of the area covered by a node's transmission range lies outside the space into which nodes are allowed to move.

Our intuitive model of Route Discovery predicted that changing to the square site, where nodes have greater degree, should both decrease containment and increase discovery cost. As Table IX shows, however, containment does decrease by $6 \%$ from $68 \%$ to $62 \%$, but the discovery cost remains relatively 
TABLE IX

SumMary of ROUTE Discovery COSTS (All-Opt DSR, SQuare Site, Constant Node Motion)

\begin{tabular}{l|c|c|c}
\cline { 2 - 4 } & Nonpropagating & Propagating & Total \\
\hline Request Og & 217 & 27 & 244 \\
\hline Request Fw & 0 & 798 & 798 \\
\hline Reply Og & 1,931 & 387 & 2,318 \\
\hline Reply Fw & 0 & 584 & 584 \\
\hline Containment & $67.0 \%$ & $18.0 \%$ & $62.0 \%$ \\
\hline Cost & 9.87 & 66.05 & 16.11 \\
\hline
\end{tabular}

unchanged. Analyzing the nonpropagating and propagating requests separately makes the reasons for the discrepancy more apparent.

The reason the overall metrics do not follow our expectations is that the increased node degrees in the square site not only increase the number of nodes that overhear each ROUTE REQUEST packet (thus increasing the cost of the request), but also increase the number of nodes that overhear source routes used by their neighbors. This causes routing information about each destination to be spread more widely throughout the network than in the rectangular site. The greater spatial distribution of routing information allows nonpropagating requests to succeed more often in the square site and prevents a greater number of the expensive propagating ROUTE REQUESTS.

In the rectangular site, $24 \%$ of the RouTE REQUESTS were propagating requests, while in the square site, only $12 \%$ of the Route Discoveries required a propagating ROUTE REQUEST. This difference makes the cost and containment metrics of nonpropagating requests dominate the overall overhead for the square site, thereby giving the appearance that the discovery cost and containment metrics are roughly equivalent in the two sites. However, Route Discovery tends to be more expensive and less well-contained in the square site.

\section{EFFECTS ON CACHE CONSISTENCY}

All routing protocols that use on-demand Route Discovery must include some kind of route caching system, since the originator of a packet cannot afford the cost of doing a Route Discovery operation for every packet it wishes to send. Once the originator discovers a route through the network, it must remember the route in some kind of cache for use in sending future packets. DSR, in particular, makes even greater use of the route cache, using it not only to cache routes for the purpose of originating packets, but also for the purpose of allowing nodes to answer ROUTE REQUESTS targeted at other nodes, as explained in Section II.

When a cache is added to the system, however, the issue arises of how stale cache data is handled. In the context of a route cache, we call a route stale if any link in the route is broken, since a packet sent using the route will encounter a forwarding error when it attempts to traverse the broken link. Removing stale data from the cache of a node originating packets is critical, since any packet the node sends with a stale route will result in a ROUTE ERROR being returned, with a reasonable chance of the packet being dropped.
Allowing nodes to use the data in their route caches to issue ROUTE REPLY packets carries even greater risk, as nodes with stale cache data may return stale, incorrect routes that will pollute both that particular Route Discovery and potentially other nodes' caches.

For the purposes of analyzing cache behavior, we label the nodes as either originator nodes or forwarder nodes. Originator nodes are those that are the sources of packets in a connection, while forwarder nodes do not themselves originate data packets, but only act as routers to carry others' communications. As described in Section III, there are 14 originator nodes (which may also serve as forwarders for other connections) and 36 forwarder nodes (which are not the originator of any connections) in the communication pattern used in our simulations. The route cache of an originator node behaves very differently from that of a forwarder node, since the originator actively invokes Route Discovery to maintain good routes to the nodes to which it sends packets, and it is the target of ROUTE ERRORS whenever it sends a packet with a broken route. Forwarder nodes must learn and correct routes opportunistically.

\section{A. Contents of the Route Cache}

The most basic measure of how well a node's route cache performs is the percentage of cache lookups that actually find a good cached route in the node's cache. This measure is a function of the percentage of good links in a node's cache and the overall cache hit rate. Fig. 8 shows the average number of links in each node's cache, broken out into number of good links and bad links, for our constant node motion scenarios. On average, $84 \%$ of the links in caches were found to be good while sampling once per second. As expected, all of the bad links in the caches are due to node motion, and there are $0 \%$ bad links in the caches of nodes in the scenarios with no node motion. Originating nodes have a slightly greater number of links in their caches, due to the fact that they receive ROUTE REPLY packets from nodes throughout the network when they perform Route Discovery, whereas forwarding nodes only hear ROUTE REPLIES that travel past them on the way to an originating node. That the difference is slight argues that Route Discovery is successfully priming the caches of the forwarding nodes.

To determine if the routes in the nodes' caches are useful, we evaluated the cache hit rate for each node in our simulations and show this data in Fig. 9. As described in Section II, nodes use their cache when originating packets, when deciding whether to return a cache reply in response to a received ROUTE REQUEST, and when salvaging. Originating nodes have a significantly higher hit rate than forwarding nodes, since the vast majority of the cache lookups that originating nodes perform are for packets to destinations for which they have performed Route Discovery. The hit rate of forwarding nodes is sensitive to both the geometry of the space and the communication pattern, since a node's hit rate will go up if the ROUTE REQUESTS that it overhears are for destinations in close spatial proximity, and down as it processes ROUTE REQUESTS for nodes far away from it. Forwarding nodes in the rectangular 


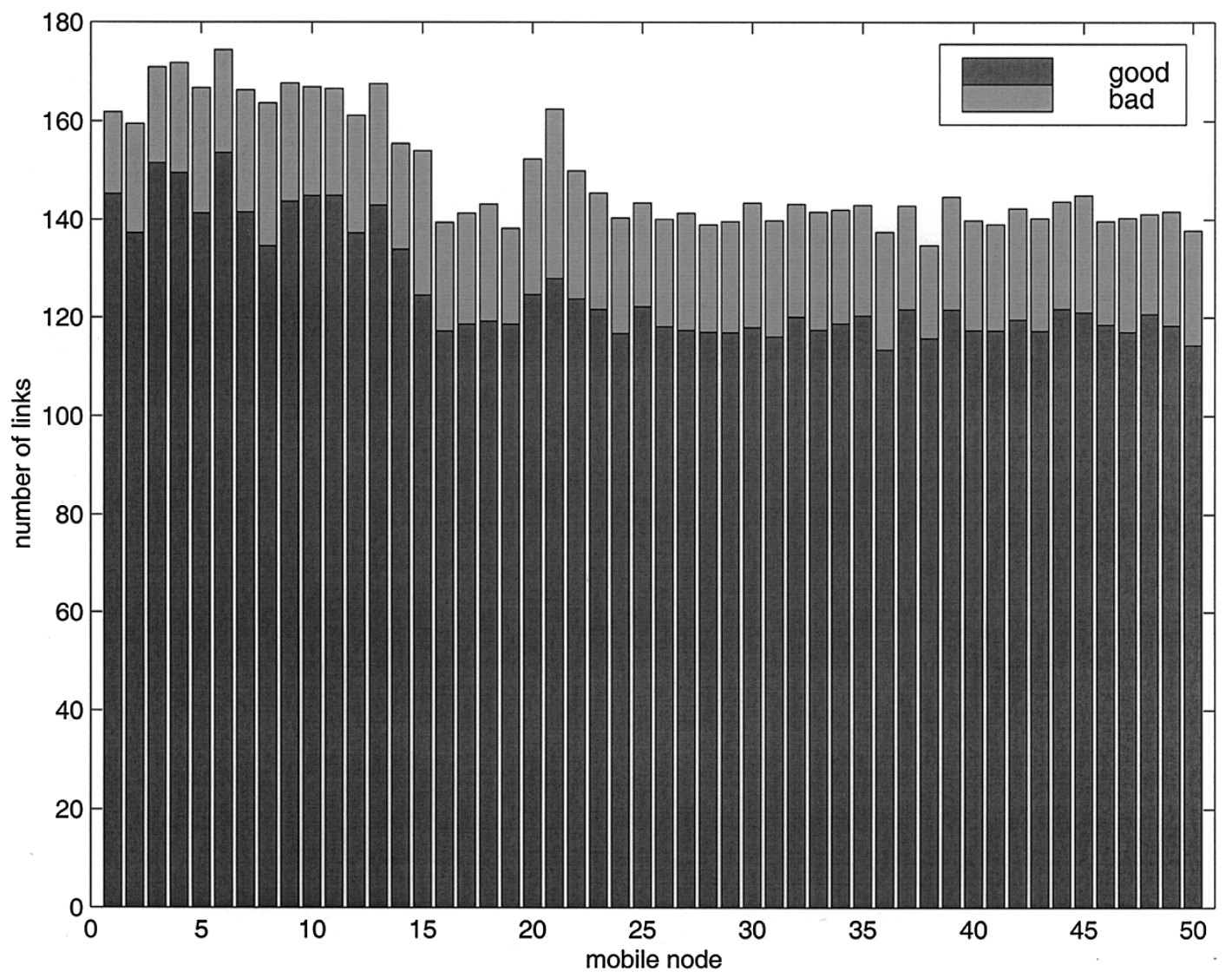

Fig. 8. Average number of links in each node's cache over simulation runs for all-opt DSR (rectangular site, constant motion). Nodes 1-14 are originating nodes.

site have an average hit rate of only $55 \%$, which, though low, is consistent with the philosophy of on-demand Route Discovery: there is no reason to have a route to a destination unless you are originating packets to it. In the square site, the average hit rate for forwarding nodes increases to $62 \%$ since nodes are fewer hops apart, and there is greater shared knowledge as nodes overhear a greater number of useful source routes that they can then add to their route caches (Section II-C).

\section{B. The Quality of Route Replies}

Nodes in the network use their caches to generate ROUTE REPLY packets in response to other nodes' Route Discoveries, in order to limit the propagation of ROUTE REQUESTS (Section II-C). Given that there may be some stale data in nodes' caches, the question arises as to the extent of the cache pollution that the Route Discovery process might cause as bad replies from cache are returned to the initiator of a Route Discovery and overheard by forwarding nodes. We collected statistics for this over our scenarios using all-opt DSR and report these results in Table X. Using the nominal $250 \mathrm{~m}$ transmission range of the radios modeled in our simulations to determine if the links are good, $40 \%$ of the ROUTE REPLIES received by the initiator of a Route Discovery contain routes that would not work if used.

That $40 \%$ of ROUTE REPLIES contain broken routes is not surprising considering how cached information is learned and why a Route Discovery is initiated. Route Discovery is performed when a node wants to send a packet to a destination to which it does not have any routes. This occurs because either the node has never had a route to the destination or because its last working route to the destination just broke, many times as the result of some major network topology change that altered the available paths to reach the destination. Since many nodes will have cached routes to that destination with links in common, the breakage of any of those links will cause many of the cached replies generated for that destination to be bad. In our simulations of all-opt DSR, the overwhelming majority of ROUTE REPLY packets are based on cached data, and only $59 \%$ of those replies carry correct routes. Though $84 \%$ of the links in the nodes' caches are good, the probability of a route being good given that it has been retrieved from a forwarding node's cache in our simulations is only $56 \%$. Even replies from the target itself are not $100 \%$ correct, since routes can change while the REPLY propagates back to the requester.

If replying from cache is disabled and only propagating Route REQUESTS are sent, a total of 8413 RouTE REPLY messages are received by initiators, of which $93 \%$ are good. While disabling the replying from cache mechanism significantly increases the number of working routes an initiator receives, it also increases overhead and latency as described in Sections V and VI.

The fact that DSR continues to have a very high packet delivery ratio (Fig. 1) even when $40 \%$ of the routes returned by Route Discovery are incorrect, argues that Route Maintenance 


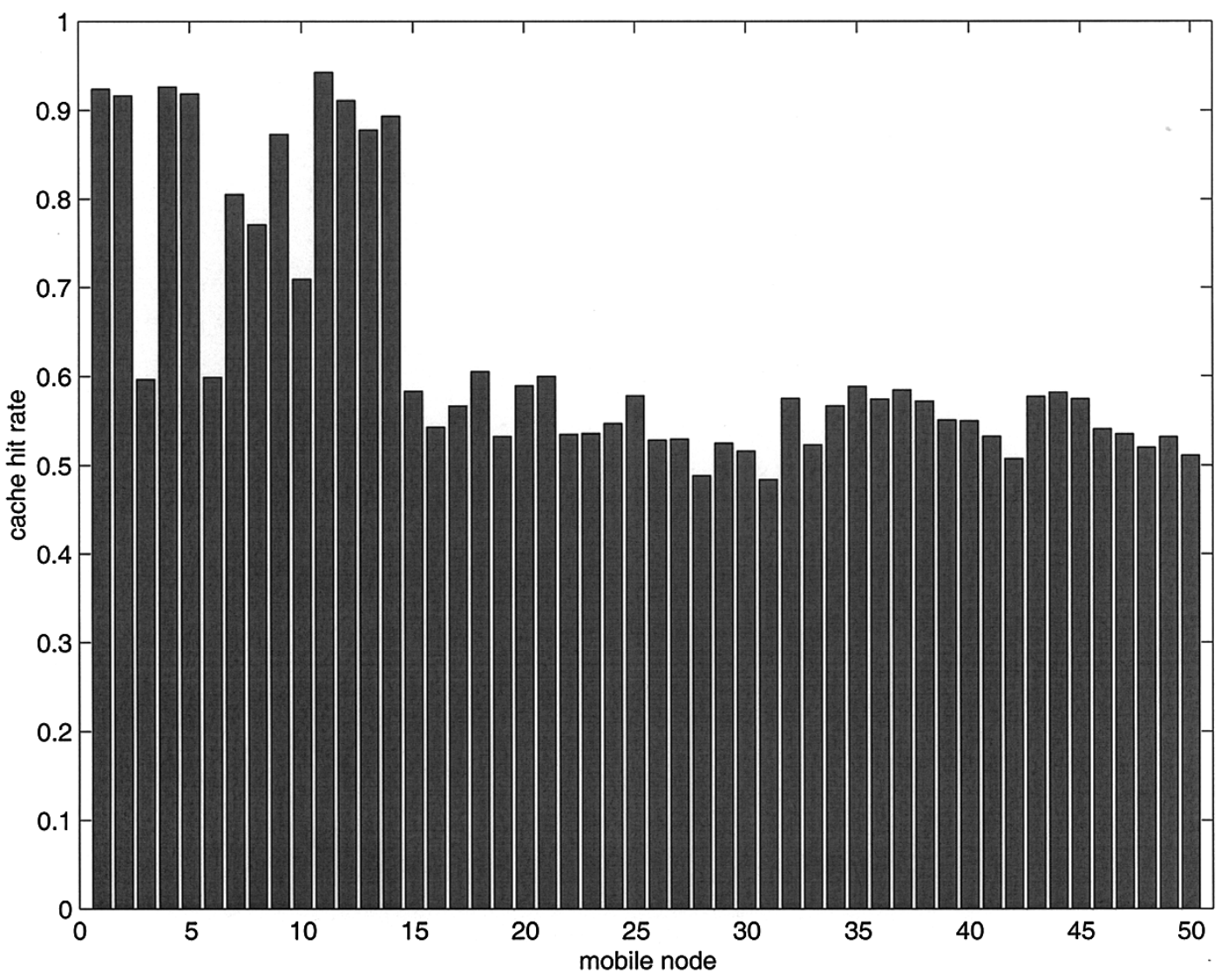

Fig. 9. Cache hit rate for all-opt DSR (rectangular site, constant motion). Nodes 1-14 are originating nodes.

TABLE X

The Correctness of Source Routes Returned by Route Discovery

\begin{tabular}{l|c|c}
\cline { 2 - 3 } & \# replies & $\%$ good \\
\hline from target & 119 & $95.2 \%$ \\
\hline from cache & 6809 & $59.4 \%$ \\
\hline total & 6928 & $60.0 \%$ \\
\hline
\end{tabular}

is performing well so that the discovery and dissemination of bad routes does not result in the continued use of bad routes.

\section{CONCLUSIONS AND FUtURE WORK}

In this paper, we have presented a detailed examination of the fine-grain performance of on-demand routing protocols. We analyzed the latency of Route Discovery, the cost of Route Discovery, and the effect of on-demand behavior on routing cache consistency, drawing our examples for study from the DSR protocol. DSR provides a good source of examples for this study, since it is based entirely on on-demand behavior, and thus our results are not affected by any periodic or background activity within the protocol.

We have identified several mechanisms that can be used to reduce the cost of Route Discovery and isolated the performance improvement due to each of these mechanisms. As expected, we found that a naive approach to Route Discovery is expensive, both in terms of latency and the number of packets that are sent to complete the discovery. However, we have shown how the twin techniques of using route caches to answer route requests and using nonpropagating requests to limit the search performed by the routing protocol can reduce the mean latency of Route Discovery from $403 \mathrm{~ms}$ to well under $40 \mathrm{~ms}$ and decrease the total overhead from 102 packets per discovery to 17 packets. Examining the role of the route cache in an on-demand routing protocol, we found that the cache is able to acquire useful information about the overall network topology solely by extracting routing information from packets that pass through and near it. Nodes were able to learn about sufficiently many destinations to achieve a cache hit rate of $55 \%$, whether the nodes were initiating Route Discovery themselves or not. Efficient Route Maintenance is critical in all systems with route caches, as we found that $16 \%$ of the links in the nodes' caches were stale and up to $41 \%$ of the ROUTE REPLIES sent based on cached data contained on broken routes. The DSR Route Maintenance techniques met the challenge however, as more than $90 \%$ of the data packets were successfully delivered even at constant node motion.

Many of the techniques and lessons learned from this work can be applied to the other on-demand routing protocols, such as AODV, TORA, and ZRP. In particular, by adding a mechanism to share routing information among nodes in the network (e.g., having a subset of the data packets each record the route it takes through the network), protocols such as these three could make greater use of their route caches to control the overhead cost of Route Discovery. 
At present, we are extending this work by exploring the effects of varying the number of nodes, the rate and pattern of node movement, and the type of communication pattern. We are also working to examine and isolate the effects of the other optimizations to DSR discussed in this paper, including gratuitous ROUTE REPLIES, salvaging, gratuitous ROUTE ERRORS, snooping, and tapping. In addition, we are presently experimenting with the idea of using a link-state cache that would allow us to combine the results of multiple Route Discoveries.

\section{REFERENCES}

[1] J. Broch, D. B. Johnson, and D. A. Maltz, "The dynamic source routing protocol for mobile ad hoc networks," Internet-Draft, draft-ietf-manetdsr-01.txt, work-in-progress, Dec. 1998.

[2] J. Broch, D. A. Maltz, D. B. Johnson, Y.-C. Hu, and J. Jetcheva, "A performance comparison of multi-hop wireless ad hoc network routing protocols," in Proc. Fourth Annual ACM/IEEE Int. Conf. Mobile Computing and Networking, Dallas, TX, Oct. 1998, pp. 85-97.

[3] M. S. Corson and A. Ephremides, "A distributed routing algorithm for mobile wireless networks," Wireless Networks, vol. 1, pp. 61-81, Feb 1995.

[4] K. Fall and K. Varadhan, Eds., " $n s$ notes and documentation." (1998). VINT Project, Univ. California, Berkeley, CA, LBL, USC/ISI, and Xerox PARC. [Online]. Available WWW: http://www-mash.cs.berkeley.edu/ns/.

[5] Z. J. Haas and M. R. Pearlman, "A new routing protocol for the reconfigurable wireless networks," in Proc. Sixth IEEE Int. Conf. Universal Personal Communications, Oct. 1997.

[6] _ "The performance of query control schemes for the zone routing protocol," in Proc. ACM SIGCOMM'98, pp. 167-177.

77] "The zone routing protocol (ZRP) for ad hoc networks," InternetDraft, draft-ietf-manet-zone-zrp-01.txt, work-in-progress, Aug. 1998.

[8] IEEE Wireless LAN Medium Access Control (MAC) and Physical Layer (PHY) Specifications, IEEE Std 802.11-1997, 1997.

[9] D. B. Johnson, "Routing in ad hoc networks of mobile hosts," in Proc. IEEE Workshop Mobile Computing Systems and Applications, Dec. 1994 pp. $158-163$

[10] D. B. Johnson and D. A. Maltz, "Dynamic source routing in ad hoc wireless networks," in Mobile Computing, T. Imielinski and H. Korth, Eds. Norwell, MA: Kluwer, ch. 5, pp. 153-181, 1996.

[11] J. Jubin and J. D. Tornow, "The DARPA packet radio network protocols," Proc. IEEE, vol. 75, pp. 21-32, Jan. 1987.

[12] V. D. Park and M. S. Corson, "A highly adaptive distributed routing algorithm for mobile wireless networks," in Proc. INFOCOM'97, pp. $1405-1413$

[13] "Temporally-ordered routing algorithm (TORA) version 1: Functional specification," Internet-Draft, draft-ietf-manet-tora-spec01.txt, work-in-progress, Aug. 1998.

[14] C. E. Perkins and P. Bhagwat, "Highly dynamic destination-sequenced distance-vector routing (DSDV) for mobile computers," in Proc. SIGCOMM'94 Conf. Communications Architectures, Protocols and Applications, pp. 234-244.

[15] C. E. Perkins and E. M. Royer, "Ad hoc on demand distance vector (AODV) Routing," Internet-Draft, draft-ietf-manet-aodv-02.txt, Nov. 1998, work-in-progress.

[16] B. Tuch, "Development of WaveLan, an ISM band wireless LAN," AT\&T Tech. J., vol. 72, pp. 27-33, July/Aug. 1993.

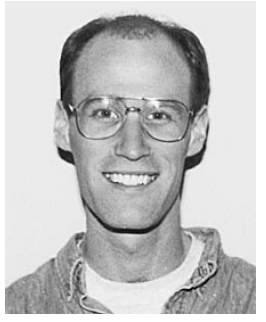

David A. Maltz received the S.B. and S.M. degrees in electrical engineering and computer science from the Massachusetts Institute of Technology, Cambridge, in 1994 . He is currently a Ph.D. candidate in the Computer Science Department at Carnegie Mellon University, Pittsburgh, PA and is supported by an Intel Graduate Fellowship. He was previously supported by an IBM Cooperative Fellowship.

He has been designing and evaluating protocols for ad hoc networks for the past six years. As an active participant in the IETF, he is working to create and standardize protocols for both Mobile IP and ad hoc networks. His other interests include computer-supported cooperative work and collaborative information filtering.

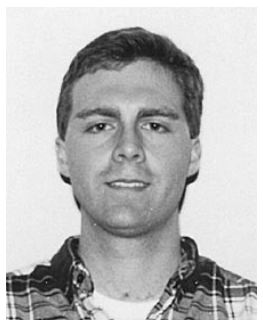

Josh Broch received the B.S. degree in mathematics and computer science from Barry University, in 1995 and the M.S. degree in information networking from Carnegie Mellon University, Pittsburgh, PA, in 1996. He is currently working towards the Ph.D degree in electrical and computer engineering at Carnegie Mellon University.

He worked for five years as a Systems Engineer at Connections for Business, Hollywood, FL and has interned at Oak Ridge National Lab and Microsoft He authored the first implementation of Mobile IP for IPv6 and is a coauthor of a leading IETF proposal for multihop routing in ad hoc wireless networks. His recent work focuses on the development and analysis of routing protocols for ad hoc networks.

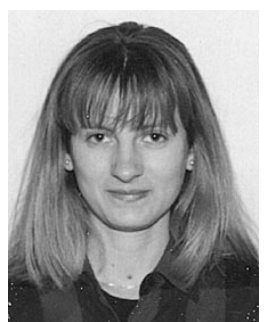

Jorjeta Jetcheva received the B.A. degree in computer science (summa cum laude) and mathematics from Mount Holyoke College, South Hadley, MA, in 1997. She is currently working towards the Ph.D degree in computer science at Carnegie Mellon University, Pittsburgh, PA.

She has been working on design, analysis, simulation and measurement of routing protocols for multihop ad hoc networks. Her research interests include ad hoc networking, mobile IP, and network performance analysis. She has interned at the DataViews Corporation, Northampton, MA, where she worked on dynamic data visualization software.

David B. Johnson (S'74-M'77), for a photograph and biography, see this issue, p. 1331. 